\title{
Combination of CRP and NLR: a better predictor of postoperative survival in patients with gastric cancer
}

This article was published in the following Dove Press journal: Cancer Management and Research

\author{
Jing Guo ${ }^{1,2, *}$ \\ Shangxiang Chen ${ }^{1,2, *}$ \\ Yongming Chen ${ }^{1,2, *}$ \\ Shun $\mathrm{Li}^{1,2}$ \\ Dazhi $X u^{1,2}$
}

'State Key Laboratory of Oncology in South China, Collaborative Innovation Center for Cancer Medicine, Guangzhou, People's Republic of China; ${ }^{2}$ Department of Gastric Surgery, Sun Yat-Sen University Cancer Center, Guangzhou, People's Republic of China

*These authors contributed equally to this work
Correspondence: Dazhi Xu

Department of Gastric Surgery,

Sun Yat-Sen University Cancer Center,

65I\# East Dongfeng Road, Guangzhou

51 0060, Guangdong Province, People's

Republic of China

Fax +86208734 3737

Email xudzh@sysucc.org.cn
Objectives: C-reactive protein (CRP) and neutrophil to lymphocyte ratio (NLR) were independent predictive factors for gastric cancer (GC). Our study was designed to prove the prognostic value of the combination of CRP and NLR (COC-NLR) in GC patients.

Materials and methods: A total of 1,058 GC patients who underwent D2 resection from Sun Yat-Sen University Cancer Center between 2003 and 2013 were included. They were divided into three groups (low: NLR $\leq 2.5, \mathrm{CRP} \leq 6.1$; medium: NLR $>2.5, \mathrm{CRP} \leq 4.5$; high: $\mathrm{NLR}>2.5$, CRP $>4.5$ or NLR $\leq 2.5$, CRP $>6.1$ ) by the random forest method. Survival analysis stratified by COC-NLR groups was performed.

Results: The mean survival time for each group was: for the low group 75.44 months (95\% CI: 72.48-78.40), the medium group 56.50 months (95\% CI: 50.68-62.31), and the high group 38.65 months (95\% CI: 34.51-42.97). The low group showed obviously better overall survival (OS) than other two groups $(p<0.001)$. Survival analysis showed that COC-NLR had statistical significance in both univariate and multivariate analyses $(p<0.01)$.

Conclusion: This study showed that COC-NLR could work as an independent prognostic factor in GC and provide more accurate prediction than single NLR or CRP.

Keywords: inflammation index, NLR, CRP, combination, prognosis, gastric cancer

\section{Introduction}

Gastric cancer (GC) is the second most common cause of cancer-related mortality worldwide, including many Asian countries. ${ }^{1,2}$ Most GC patients are diagnosed at advanced stage and have poor survival. As a result, there is a need to obtain an accurate, costless, and easily accessible marker to evaluate the prognosis of curative resection GC patients.

In clinical practice, many studies reported that the systemic inflammatory response was associated with postoperative survival of patients with several types of cancer, such as gastric, lung, colorectal, and head and neck cancer. ${ }^{3-6}$ Recently, many markers of inflammatory response, including neutrophil to lymphocyte ratio (NLR), platelet to lymphocyte ratio (PLR), C-reactive protein (CRP), albumin (Alb), globulin, and the Glasgow Prognostic Score (GPS) were demonstrated as independent predictive factors of GC. ${ }^{7-12}$ In addition, some kinds of the combined markers were also studied in other cancers. Zhang et al and Ishizuka et al reported that the combination of preoperative platelet count and NLR was able to predict the prognosis of patients and direct clinical treatment with lung cancer and colorectal cancer, respectively. ${ }^{13,14}$ Huang et al found that the combination of CRP and carcinoembryonic antigen was superior to CRP or 
CEA as a more precise prognostic factor in patients with esophageal carcinoma. ${ }^{15}$ However, the role of combination of these inflammatory biomarkers in accessing GC prognosis is less well-known. We conducted a systematic literature review and surprisingly found that the significance of the combination of CRP and NLR (COC-NLR) for GC had not been studied yet. Therefore, we collected related data and designed this retrospective study to explore the relationship between COC-NLR and the prognosis of GC patients.

\section{Materials and methods}

\section{Study population and data collection}

A retrospective review was performed by using a database of 1,058 GC patients who had undergone D2 gastrectomy with R0 resection between December 2003 and January 2013 in Cancer Center of Sun Yat-Sen University Cancer Center (Guangzhou, China). The inclusion criteria were as follows: 1) patients were confirmed as stage I-III histologically; 2) patients underwent D2 gastrectomy with R0 resection; 3) patients whose number of lymph nodes retrieved were no less than 15 ; 4) no acute or chronic inflammation, immune disease, hematological disease, liver disease, or concomitant cancer that could influence the level of the biomarkers; 5) no neoadjuvant chemotherapy or radiotherapy; 6) completed follow-up data.

Various potential prognostic factors were investigated, including age, sex, preoperative blood variables, metastatic lymph node ratio, tumor location, histological type, and tumor-node-metastasis (TNM) stage (American Joint Committee on Cancer criteria, AJCC criteria 7th edition). ${ }^{16-18}$ Data were obtained from our hospital cancer registry. All patients were followed up regularly until December 2016 or until death or the day of cancer recurrence. The median follow-up period was 35 months. Overall survival (OS) was calculated from the date of surgery to the date of death or last follow-up.

The Alb globulin ratio (AGR) is calculated by measured total protein, measured Alb, and calculated globulin (total protein - Alb). ${ }^{7,19}$ The NLR and PLR were defined as the absolute neutrophil count or platelet count divided by the absolute lymphocyte count, respectively. ${ }^{20}$ The systemic immune inflammation index (SII) was calculated as follows: $\mathrm{SII}=\mathrm{P} \times \mathrm{N} / \mathrm{L}(\mathrm{P}$ : platelet counts, $\mathrm{N}$ : neutrophil counts, $\mathrm{L}$ : lymphocyte counts). ${ }^{21}$ Using standard thresholds $(>10 \mathrm{mg} / \mathrm{L}$ for CRP and $<35 \mathrm{~g} / \mathrm{L}$ for Alb), the GPS was calculated by Alb and CRP. The CRP/Alb ratio was defined as the ratio of preoperative serum CRP level divided by the serum Alb level. ${ }^{22}$

The study was approved by the Research Ethics Committee of Sun Yat-Sen University Cancer Center. Written informed consent was obtained from all individual participants included in the study.

\section{Random forest analysis}

Breiman et al proposed random forests, which added an additional layer of randomness to bagging. In addition to constructing each tree using a different bootstrap sample of the data, random forests change how the classification and regression trees are constructed. ${ }^{23} \mathrm{R}$ Statistical Software was used to discover the optimal cutoff points of the NLR and CRP based on the outcome. The random forest algorithm package was used to evaluate the levels of the NLR and CRP (Figure 1).

\section{Statistical analysis}

$\chi^{2}$ test was used for categorical variables. The optimal cutoff values were obtained by the Youden index. ${ }^{24}$ Variables proved to be statistically significant in the univariate analysis were included into the Cox multivariable analysis. The discriminatory ability of the factors to predict survival time was assessed using the area under the curve (AUC) method. Kaplan-Meier curve was used for survival analysis.

When the $p$ value was $<0.05$, the result was statistically significant. All statistical analyses were performed by the software statistical package for social sciences version 19.0 (IBM Corporation, Armonk, NY, USA) and the R software version 3.13 (http://www.r-project.org/).

\section{Results}

Based on the cutoff values, 1,058 patients were classified into three groups (low: NLR $\leq 2.5, \mathrm{CRP} \leq 6.1$; medium: NLR $>2.5, \mathrm{CRP} \leq 4.5$; high: NLR $>2.5$, CRP $>4$ or NLR $\leq 2.5, \mathrm{CRP}>6.1$ ). The three groups had 581, 187, and 290 patients, respectively. The relationship between COC-NLR and clinicopathological characteristics are shown in Table 1. In the three groups, no significant correlations were found in sex, level of lymphocytes, and Alb. There were significant differences among the three groups, including age, neutrophils, platelet, CRP, AGR, NLR, PLR, SII, GPS, modified GPS (mGPS), high-sensitivity mGPS (HS-mGPS), CRP/Alb ratio, tumor location, tumor size, metastatic lymph node ratio, histological type, and TNM stage.

Associations between each variable and OS were presented in Table 2, in which univariate analysis and multivariate Cox regression were performed. Significant variables (age, neutrophils, platelets, CRP, AGR, NLR, PLR, SII, GPS, mGPS, HS-mGPS, CRP/Alb ratio, tumor location, tumor size, metastatic lymph node ratio, histological type, and 


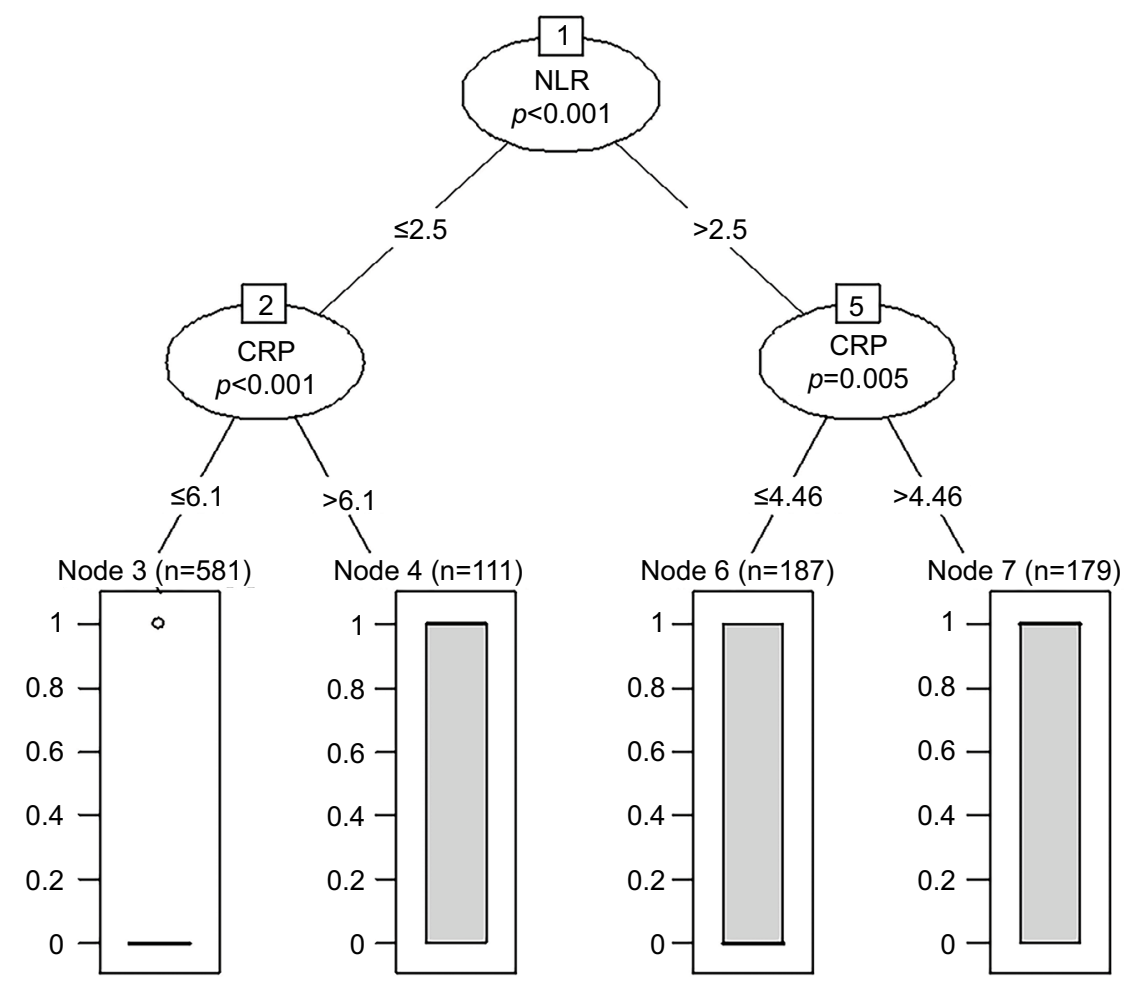

Figure I Random forest analysis for the optimal cutoff points.

Abbreviations: NLR, neutrophil to lymphocyte ratio; CRP, C-reactive protein.

TNM stage) in the univariate analysis were included in the multivariate Cox proportional hazard model. The multivariate analysis indicated that age, neutrophils, CRP, AGR, HSmGPS, tumor location, tumor size, metastatic lymph node ratio, histological type, and TNM stage were independent prognostic factors for survival time of patients.

The predictive values of independent factors (neutrophils, CRP, AGR, and HS-mGPS) were assessed by the receiver operating characteristic (ROC) curve method. With the highest AUC ( 1 year: 0.662, 95\% CI: 0.609-0.715, $p<0.01 ; 3$ years: $0.644,95 \%$ CI: $0.611-0.677, p<0.01 ; 5$ years: $0.655,95 \%$ CI: $0.614-0.696, p<0.01$ ), the COC-NLR had the optimal discrimination ability which clearly showed that it is superior to other inflammatory markers (Figure 2, Table 3).

We used Kaplan-Meier curves to assess the survival time of patients in different groups. The mean OS of the high, medium, and low groups was 38.7 (95\% CI: 21.3-27.1), 56.5 (95\% CI: 50.7-62.3), and 75.4 (95\% CI: 72.5-78.4) months, respectively (Figure 3A). Because GC patients of stage I always had good prognosis, we performed subgroup analysis in stage II (Figure 3B) and stage III (Figure 3C). We found patients in the low group had better survival than the medium group and the high group.

\section{Discussion}

In this report, we first proved that the preoperative COC-NLR was associated with the prognosis of GC patients. Moreover, COC-NLR was a better prognostic factor compared to use CRP or NLR only. The high group of the preoperative COCNLR was associated with adverse survival probabilities in GC.

Indeed, as the important markers of inflammation response, the prognostic values of CRP and NLR have been given great concern. Nozoe et al found that GC patients with preoperative CRP elevation usually had shorter survival time. ${ }^{25}$ Lee et al and Sun et al reported that high level of NLR predicted poor outcome of GC patients. Besides, the measurement of NLR may be used for personalized cancer care in the future. ${ }^{26,27}$ Interestingly, our results were in accordance with the previous studies. ${ }^{25-27}$

Survival analysis showed that CRP has statistical significance in both univariate $(p<0.01)$ and multivariate $(p<0.01)$ analyses. But NLR has statistical significance in univariate analysis only $(p<0.01)$. The group with lower level of CRP also exhibited better survival than the higher group, which indicated that CRP was a negative factor for the prognosis of GC. However, the ROC curve showed that COC-NLR could provide more accurate prediction than any other conventional inflammatory markers in this study, including single CRP. Meanwhile, it was also verified in our subgroup survival analysis. 
Table I Relationship between COC-NLR and clinicopathologic characteristics

\begin{tabular}{|c|c|c|c|c|}
\hline Variable & $\begin{array}{l}\text { Low } \\
(n=58 I)\end{array}$ & $\begin{array}{l}\text { Medium } \\
(n=187)\end{array}$ & $\begin{array}{l}\text { High } \\
(n=290)\end{array}$ & $p$-value \\
\hline \multicolumn{5}{|l|}{ Patient-related factors } \\
\hline Sex & & & & 0.228 \\
\hline Male & 381 & 130 & 206 & \\
\hline Female & 200 & 57 & 84 & \\
\hline Age (years) & & & & 0.019 \\
\hline$<65$ & 421 & 129 & 183 & \\
\hline$\geq 65$ & 160 & 58 & 107 & \\
\hline Neutrophils $\left(\times 10^{9} / \mathrm{L}\right)$ & & & & $<0.01$ \\
\hline$<3.61$ & 382 & 40 & 60 & \\
\hline$\geq 3.6 \mathrm{I}$ & 119 & 147 & 230 & \\
\hline Lymphocytes (×109/L) & & & & 0.238 \\
\hline$<3.56$ & 581 & 155 & 244 & \\
\hline$\geq 3.56$ & 0 & 32 & 46 & \\
\hline Platelets ( $\left.\times 10^{9} / \mathrm{L}\right)$ & & & & $<0.01$ \\
\hline$<315.5$ & 511 & 157 & 206 & \\
\hline$\geq 315.5$ & 70 & 30 & 84 & \\
\hline CRP (mg/L) & & & & $<0.01$ \\
\hline$<4.29$ & 546 & 183 & 0 & \\
\hline$\geq 4.29$ & 35 & 4 & 290 & \\
\hline Albumin $(\mathrm{g} / \mathrm{L})$ & & & & 0.166 \\
\hline$<48.45$ & 566 & 183 & 288 & \\
\hline$\geq 48.45$ & 15 & 4 & 2 & \\
\hline AGR & & & & 0.03 \\
\hline$<1.09$ & 6 & 3 & 13 & \\
\hline$\geq 1.09$ & 575 & 184 & 277 & \\
\hline NLR & & & & $<0.01$ \\
\hline$<2.5$ & 581 & 155 & 244 & \\
\hline$\geq 2.5$ & 0 & 32 & 46 & \\
\hline PLR & & & & $<0.01$ \\
\hline$<|25.3|$ & 358 & 45 & 97 & \\
\hline$\geq|25.3|$ & 223 & 142 & 193 & \\
\hline SII & & & & $<0.01$ \\
\hline$<521.56$ & 457 & 24 & 82 & \\
\hline$\geq 521.56$ & 124 & 163 & 208 & \\
\hline GPS & & & & $<0.01$ \\
\hline 0 & 561 & 176 & 112 & \\
\hline 1 & 20 & 11 & 132 & \\
\hline 2 & 0 & 0 & 46 & \\
\hline mGPS & & & & $<0.01$ \\
\hline 0 & 505 & 155 & 6 & \\
\hline I & 72 & 32 & 174 & \\
\hline 2 & 4 & 0 & 110 & \\
\hline HS-mGPS & & & & $<0.01$ \\
\hline 0 & 476 & 142 & 0 & \\
\hline I & 99 & 44 & 232 & \\
\hline 2 & 6 & I & 58 & \\
\hline CRP/Alb ratio & & & & $<0.01$ \\
\hline$<0.096$ & 581 & 187 & 323 & \\
\hline$\geq 0.096$ & 0 & 0 & 58 & \\
\hline \multicolumn{5}{|l|}{ Tumor-related factors } \\
\hline Tumor location & & & & $<0.01$ \\
\hline Lower third & 260 & 70 & 87 & \\
\hline Middle third & $|2|$ & 35 & 50 & \\
\hline Upper third & 200 & 82 & 153 & \\
\hline
\end{tabular}

(Continued)
Table I (Continued)

\begin{tabular}{lllll}
\hline Variable & $\begin{array}{l}\text { Low } \\
(\mathbf{n = 5 8})\end{array}$ & $\begin{array}{l}\text { Medium } \\
(\mathbf{n = 1 8 7})\end{array}$ & $\begin{array}{l}\text { High } \\
(\mathbf{n = 2 9 0 )})\end{array}$ & p-value \\
\hline $\begin{array}{l}\text { Tumor size }(\mathrm{cm}) \\
<2.9\end{array}$ & 165 & 35 & 27 & $<\mathbf{0 . 0 1}$ \\
$\geq 2.9$ & 416 & 152 & 263 & \\
$\begin{array}{l}\text { Histological type } \\
\quad \text { Poor-differentiated }\end{array}$ & 366 & 99 & 196 & $<\mathbf{0 . 0 1}$ \\
adenocarcinoma & & & & \\
Other & 215 & 88 & 94 & \\
$\begin{array}{l}\text { Metastatic lymph node ratio } \\
<0.18\end{array}$ & 368 & 105 & 119 & $<\mathbf{0 . 0 1}$ \\
$\geq 0.18$ & 213 & 82 & 171 & \\
TNM stage & & & & $<\mathbf{0 . 0 1}$ \\
IA & 82 & 21 & 9 & \\
IB & 52 & 20 & 11 & \\
IIA & 58 & 20 & 11 & \\
IIB & 102 & 25 & 43 & \\
IIIA & 69 & 16 & 37 & \\
IIIB & 109 & 42 & 64 & \\
IIIC & 109 & 43 & 115 &
\end{tabular}

Notes: Low: NLR $\leq 2.5$, CRP $\leq 6$. I; medium: NLR $>2.5$, CRP $\leq 4.6$; high: NLR $>2.5$, $C R P>4$ or NLR $\leq 2.5, C R P>6$. I. Bold figures represent as statistically significant, $p<0.05$.

Abbreviations: NLR, neutrophil to lymphocyte ratio; CRP, C-reactive protein; COC-NLR, combination of CRP and NLR; AGR, albumin globulin ratio; GPS, Glasgow Prognostic Score; mGPS, modified Glasgow Prognostic Score; HS-mGPS, high-sensitivity modified Glasgow Prognostic Score; TNM, tumor-node-metastasis; Alb, albumin; PLR, platelet to lymphocyte ratio; SIl, systemic immune inflammation index.

As a relatively simple, convenient, and cheap model, COC-NLR is easy to measure in clinical practice. D2 gastrectomy is the cornerstone of treatment for patients suffering from localized GC. ${ }^{28}$ However, GC metastases early via blood, lymphatic system, and peritoneum. Even after R0 resection, almost $40 \%$ of GC patients relapse within 2 years after gastrectomy. The median OS after recurrence is as low as 7.4 months in patients with distant metastases and 10.4 months in patients with local recurrence. ${ }^{29}$ Therefore, as an accurate marker, COC-NLR can help us to predict recurrence ahead and make decisions in the management of GC patients, including selection of adjuvant therapies and determining follow-up arrangements. According to our study, high group had the poorest prognosis. For this group of patients, more powerful adjuvant chemotherapy may be used to prevent recurrence and prolong survival postoperatively. As generally known, there is strong linkage between inflammation and chemotherapy. Short survival time is always related to low chemosensitivity. Therefore, more frequent follow-up should be performed postoperatively for patients with high-risk recurrence in order to choose suitable therapies.

As an effective biomarker, COC-NLR could predict the prognosis of GC patients better than many other wellestablished systemic inflammation-based prognostic markers. 
Table 2 Hazard ratios of baseline characteristics for all-cause mortality in gastric cancer patients (univariate and multivariate analyses)

\begin{tabular}{|c|c|c|c|c|}
\hline & $\begin{array}{l}\text { Univariate analysis } \\
\text { HR }(95 \% \mathrm{Cl})\end{array}$ & $p$-value & $\begin{array}{l}\text { Multivariate analysis } \\
\text { HR }(95 \% \mathrm{Cl})\end{array}$ & $p$-value \\
\hline \multicolumn{5}{|l|}{ Patient-related factors } \\
\hline Sex (male/female) & $1.019(0.825-1.257)$ & 0.863 & & \\
\hline Age (years) $(<65 / \geq 65)$ & $1.514(1.234-1.857)$ & $<0.01$ & $1.604(1.289-1.997)$ & $<0.01$ \\
\hline COC-NLR (ref: low) & & $<0.01$ & & $<0.01$ \\
\hline Medium & $1.863(1.403-2.474)$ & & $1.693(1.195-2.397)$ & $<0.01$ \\
\hline High & $4.223(3.382-5.27 I)$ & & $2.698(I .504-4.84 I)$ & $<0.01$ \\
\hline Neutrophils $\left(\times 10^{9} / \mathrm{L}\right)(<3.6 \mathrm{I} / \geq 3.6 \mathrm{I})$ & $2.075(1.684-2.558)$ & $<0.01$ & I.37I (I.052-I.785) & 0.02 \\
\hline Lymphocytes $\left(\times 10^{9} / \mathrm{L}\right)(<3.56 / \geq 3.56)$ & $0.927(0.4 \mid 4-2.077)$ & 0.855 & & \\
\hline Platelet $\left(\times 10^{9} / \mathrm{L}\right)$ & $1.422(1.117-1.810)$ & 0.004 & I. $105(0.822-1.485)$ & 0.51 \\
\hline \multicolumn{5}{|l|}{$(<3|5.5 / \geq 3| 5.50)$} \\
\hline $\operatorname{CRP}(\mathrm{mg} / \mathrm{L})(<4.29 / \geq 4.29)$ & $3.265(2.676-3.983)$ & $<0.01$ & $1.149(0.607-2.176)$ & $<0.01$ \\
\hline Albumin $(\mathrm{g} / \mathrm{L})(<48.45 / \geq 48.45)$ & $\mathrm{I} .459(0.82 \mathrm{I}-2.592)$ & 0.198 & & \\
\hline AGR $(<2.5 / \geq 2.5)$ & $0.210(0.169-0.262)$ & $<0.01$ & $2.847(1.33|-6.09|)$ & $<0.01$ \\
\hline $\operatorname{NLR}(<2.5 / \geq 2.5)$ & $0.210(0.169-0.262)$ & $<0.01$ & $1.345(0.965-1.875)$ & 0.08 \\
\hline $\operatorname{PLR}(<|25.3||\geq| 25.3 \mid)$ & $1.443(1.181-1.763)$ & $<0.01$ & $1.105(0.822-1.485)$ & 0.51 \\
\hline SII $(<521.56 / \geq 521.56)$ & $1.762(1.444-2.15 \mathrm{I})$ & $<0.01$ & $0.795(0.573-1.103)$ & 0.17 \\
\hline GPS (ref: 0) & & $<0.01$ & & 0.21 \\
\hline 1 & $2.297(1.808-2.919)$ & $<0.01$ & $0.868(0.618-1.219)$ & 0.42 \\
\hline 2 & $3.689(2.55 I-5.336)$ & $<0.01$ & $0.443(0.179-1.097)$ & 0.08 \\
\hline mGPS (ref: 0) & & $<0.01$ & & 0.14 \\
\hline 1 & $0.312(0.235-0.4 \mid 4)$ & $<0.01$ & $1.052(0.709-1.559)$ & 0.80 \\
\hline 2 & $0.762(0.569-1.020)$ & 0.068 & $0.689(0.386-1.229)$ & 0.21 \\
\hline HS-mGPS (ref: 0) & & $<0.01$ & & 0.02 \\
\hline 1 & $2.579(2.09 I-3.18 I)$ & $<0.01$ & $0.987(0.668-1.459)$ & 0.74 \\
\hline 2 & $4.124(2.944-5.775)$ & $<0.01$ & $2.764(1.242-6.150)$ & 0.06 \\
\hline CRP/Alb ratio $(<0.096 / \geq 0.096)$ & $2.945(2.076-4.179)$ & $<0.01$ & 1.501 (0.9I0-2.477) & 0.11 \\
\hline \multicolumn{5}{|l|}{ Tumor-related factors } \\
\hline Tumor location (ref: lower) & & $<0.01$ & & $<0.01$ \\
\hline Middle & $1.4 \mid 2(1.05 \mid-1.895)$ & 0.022 & $1.312(0.970-1.776)$ & 0.08 \\
\hline Upper & $2.453(1.946-3.092)$ & $<0.01$ & $1.813(1.417-2.319)$ & $<0.01$ \\
\hline Tumor size $(\mathrm{cm})(<2.9 / \geq 2.9)$ & $3.05 I(2.207-4.217)$ & $<0.01$ & $0.933(0.65 I-1.338)$ & 0.71 \\
\hline Histological type & $1.365(1.104-1.687)$ & $<0.01$ & $0.718(0.575-0.897)$ & $<0.01$ \\
\hline \multicolumn{5}{|l|}{ (ref: poor-differentiated adenocarcinoma) } \\
\hline Metastatic lymph node ratio $(<0.18 / \geq 0.18)$ & $4.753(3.817-5.919)$ & $<0.01$ & $2.099(1.526-2.888)$ & $<0.01$ \\
\hline TNM stage (ref: IA) & & $<0.01$ & & $<0.01$ \\
\hline IB & $0.037(0.015-0.091)$ & $<0.01$ & $1.625(0.513-5.147)$ & 0.41 \\
\hline IIA & $0.072(0.034-0.153)$ & $<0.01$ & $3.153(1.094-9.089)$ & 0.03 \\
\hline IIB & $0.129(0.072-0.231)$ & $<0.01$ & $4.765(1.833-12.389)$ & 0.01 \\
\hline IIIA & $0.235(0.167-0.331)$ & $<0.01$ & $5.992(2.286-15.710)$ & $<0.01$ \\
\hline IIIB & $0.377(0.269-0.527)$ & $<0.01$ & $7.352(2.807-19.254)$ & $<0.01$ \\
\hline IIIC & $0.63 \mathrm{I}(0.497-0.802)$ & $<0.01$ & 9.639 (3.620-25.669) & $<0.01$ \\
\hline
\end{tabular}

Note: Bold figures represent as statistically significant, $p<0.05$.

Abbreviations: NLR, neutrophil to lymphocyte ratio; CRP, C-reactive protein; COC-NLR, combination of CRP and NLR; AGR, albumin globulin ratio; GPS, Glasgow Prognostic Score; mGPS, modified Glasgow Prognostic Score; HS-mGPS, high-sensitivity modified Glasgow Prognostic Score; TNM, tumor-node-metastasis; Alb, albumin; PLR, platelet to lymphocyte ratio; SIl, systemic immune inflammation index; HR, hazard ratio; Ref, reference.

Based on such a large cohort study, our results were reliable. We supposed that the potential mechanism may be as follows. Recently, some studies ${ }^{30-32}$ demonstrated that the survival of cancer patients was determined by tumor-related factors and host-related factors simultaneously. Cancer-related inflammation can affect cell proliferation, invasion, metastasis, cell survival, tumor-cell migration, and angiogenesis. On the one hand, inflammation contributed to tumorigenesis. On the other hand, the tumor itself could release inflammatory mediators and cause inflammatory response. ${ }^{30-32}$

As a valuable classification algorithm, random forest has been used in many fields, including gene selection, classification of microarray data, prediction of protein-protein interactions, and so on. ${ }^{33,34}$ Our study first used the method to find out the cutoff points of two biomarkers, which helped us to perform a more powerful and accurate data processing. 

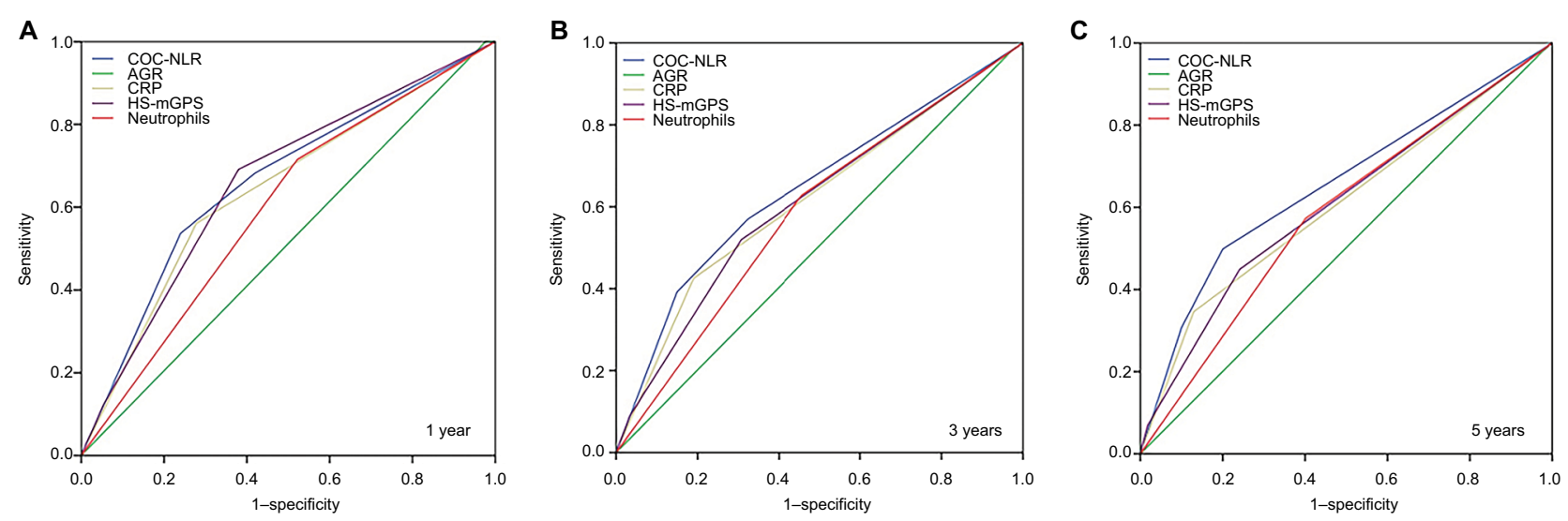

Figure 2 (A-C) The predictive abilities of the inflammatory markers were compared by receiver operating characteristic curves for I, 3 , and 5 years.

Abbreviations: NLR, neutrophil to lymphocyte ratio; CRP, C-reactive protein; COC-NLR, combination of CRP and NLR; AGR, albumin globulin ratio; HS-mGPS, highsensitivity modified Glasgow Prognostic Score.

Table 3 Comparison of the AUCs for the five inflammatory markers

\begin{tabular}{llll}
\hline & AUC & $95 \% \mathbf{C l}$ & p-value \\
\hline I year & & & \\
AGR & 0.512 & $0.458-0.565$ & 0.671 \\
Neutrophils & 0.597 & $0.545-0.648$ & $<0.01$ \\
HS-mGPS & 0.660 & $0.609-0.711$ & $<0.01$ \\
CRP & 0.641 & $0.587-0.695$ & $<0.01$ \\
COC-NLR & 0.662 & $0.609-0.715$ & $<0.01$ \\
3 years & & & \\
AGR & 0.504 & $0.470-0.539$ & 0.807 \\
Neutrophils & 0.586 & $0.552-0.620$ & $<0.01$ \\
HS-mGPS & 0.611 & $0.577-0.645$ & $<0.01$ \\
CRP & 0.618 & $0.584-0.651$ & $<0.01$ \\
COC-NLR & 0.644 & $0.611-0.677$ & $<0.01$ \\
$\mathbf{5}$ years & & & \\
AGR & 0.502 & $0.454-0.549$ & 0.946 \\
Neutrophils & 0.586 & $0.540-0.633$ & $<0.01$ \\
HS-mGPS & 0.609 & $0.565-0.652$ & $<0.01$ \\
CRP & 0.608 & $0.506-0.651$ & $<0.01$ \\
COC-NLR & 0.655 & $0.614-0.696$ & $<0.01$ \\
\hline
\end{tabular}

Abbreviations: NLR, neutrophil to lymphocyte ratio; CRP, C-reactive protein; COC-NLR, combination of CRP and NLR; AGR, albumin globulin ratio; HS-mGPS, high-sensitivity modified Glasgow Prognostic Score; AUC, area under the curve.
However, our study still had some limitations. First, it was a retrospective and single-institution study, which needs further validation in the future. Second, our result cannot be used to assess the prognosis of stage IV GC patients.

It is well-known that CRP and NLR are easy to measure routinely. As a novel combination of these two significant prognostic factors, COC-NLR is better in predicting the prognosis and indicating the treatment for $\mathrm{GC}$ patients.

\section{Acknowledgments}

All work was completed at the Department of Gastric Surgery, Sun Yat-Sen University Cancer Center, Guangzhou, People's Republic of China. We confirm that this paper has not been published or contributed to other journals.

\section{Author contributions}

All authors contributed toward data analysis, drafting and revising the paper and agree to be accountable for all aspects of the work.
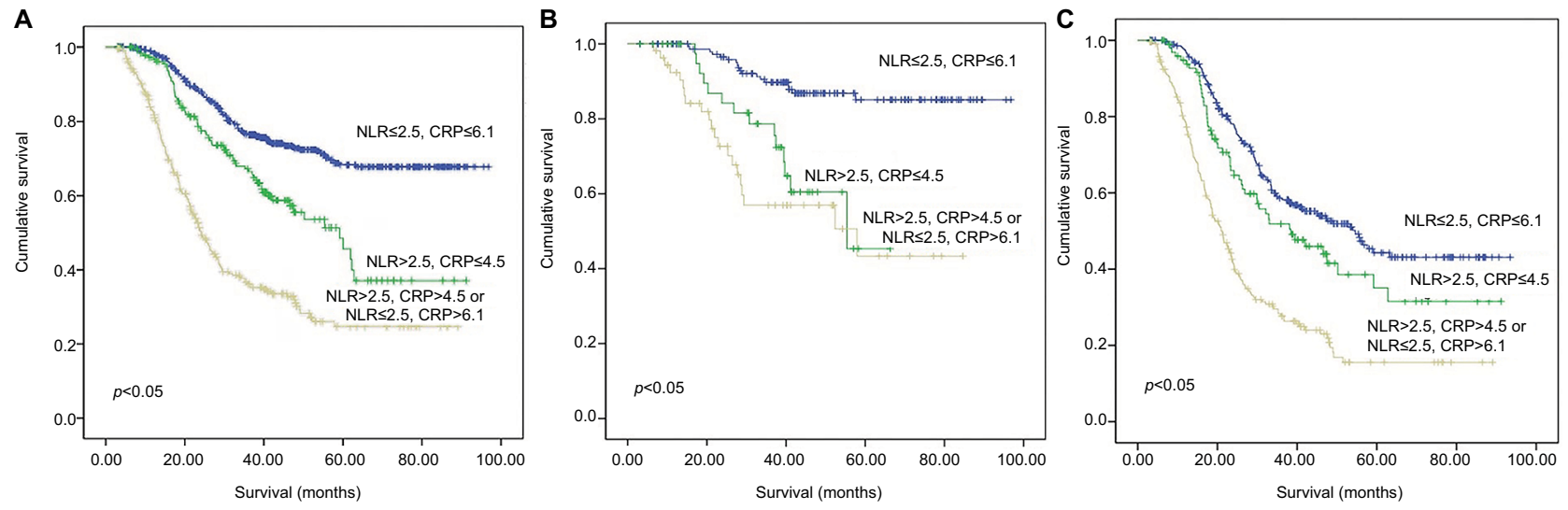

Figure 3 (A) The optimal survival curves of all patients according to the different levels of COC-NLR. (B) The optimal survival curves of stage II patients according to the different levels of COC-NLR. (C) The optimal survival curves of stage III patients according to the different levels of COC-NLR.

Abbreviations: NLR, neutrophil to lymphocyte ratio; CRP, C-reactive protein; COC-NLR, combination of CRP and NLR. 


\section{Disclosure}

The authors report no conflicts of interest in this work.

\section{References}

1. Bang YJ, Kim YW, Yang HK, et al. Adjuvant capecitabine and oxaliplatin for gastric cancer after D2 gastrectomy (CLASSIC): a phase 3 open-label, randomised controlled trial. Lancet. 2012;379(9813): 315-321.

2. Leung WK, Wu MS, Kakugawa $Y$, et al. Screening for gastric cancer in Asia: current evidence and practice. Lancet Oncol. 2008;9(3): 279-287.

3. Gao Y, Huang D. The value of the systematic inflammation-based Glasgow Prognostic Score in patients with gastric cancer: a literature review. J Cancer Res Ther. 2014;10(4):799-804.

4. Scott HR, Mcmillan DC, Forrest LM, Brown DJF, Mcardle CS, Milroy R. The systemic inflammatory response, weight loss, performance status and survival in patients with inoperable non-small cell lung cancer. $\mathrm{Br}$ J Cancer. 2002;87(3):264-267.

5. Roxburgh CSD, Mcmillan DC. Cancer and systemic inflammation: treat the tumour and treat the host. Br J Cancer. 2014;110(6):1409-1412.

6. Rassouli A, Saliba J, Castano R, Hier MP, Zeitouni A. Systemic inflammatory markers as independent prognosticators of head and neck squamous cell carcinoma. Head Neck. 2015;37(1):103-110.

7. Kibrick AC, Clements AB. A comparative study of the serum albumin-globulin ratio, the cephalin-cholesterol flocculation, and the thymol turbidity tests for liver function. J Lab Clin Med. 1948;33(6): 62-671.

8. Wang D, Yang JX, Cao DY, et al. Preoperative neutrophil-lymphocyte and platelet-lymphocyte ratios as independent predictors of cervical stromal involvement in surgically treated endometrioid adenocarcinoma. Onco Targets Ther. 2013;6:211-216.

9. Yodying H, Matsuda A, Miyashita M, et al. Prognostic significance of neutrophil-to-lymphocyte ratio and platelet-to-lymphocyte ratio in oncologic outcomes of esophageal cancer: a systematic review and meta-analysis. Ann Surg Oncol. 2016;23(2):1-9.

10. Yang X, Huang Y, Feng JF, Liu JS. Prognostic significance of neutrophilto-lymphocyte ratio in esophageal cancer: a meta-analysis. Onco Targets Ther. 2015;8:789.

11. Xinji Z, Yonggang L, Xiaojun S, Xiaowu C, Dong Z, Dajian Z. The prognostic role of neutrophils to lymphocytes ratio and platelet count in gastric cancer: a meta-analysis. Int J Surg. 2015;21:84-91.

12. Beamish AJ, Chan DS, Blake PA, Karran A, Lewis WG. Systematic review and meta-analysis of enhanced recovery programmes in gastric cancer surgery. Int J Surg. 2015;19(5 Suppl 1):46-54.

13. Zhang H, Zhang L, Zhu K, et al. Prognostic significance of combination of preoperative platelet count and neutrophil-lymphocyte ratio (COP-NLR) in patients with non-small cell lung cancer: based on a large cohort study. PLoS One. 2015;10(5):e0126496.

14. Ishizuka M, Nagata H, Takagi K, Iwasaki Y, Kubota K. Combination of platelet count and neutrophil to lymphocyte ratio is a useful predictor of postoperative survival in patients with colorectal cancer. Br J Cancer. 2013;109(2):401-407.
15. Huang Y, Liu J, Feng J. The combination of preoperative serum C-reactive protein and carcinoembryonic antigen is a useful prognostic factor in patients with esophageal squamous cell carcinoma: a combined ROC analysis. Onco Targets Ther. 2015;8:795-803.

16. Washington $\mathrm{K}$. 7th edition of the AJCC cancer staging manual: stomach. Ann Surg Oncol. 2010;17(12):3077-3079.

17. Siewert JR, Böttcher K, Stein HJ, Roder JD. Relevant prognostic factors in gastric cancer: ten-year results of the German Gastric Cancer Study. Ann Surg. 1998;228(4):449-461.

18. Wang SY, Yeh CN. Clinical impact of positive surgical margin status on gastric cancer patients undergoing gastrectomy. Ann Surg Oncol. 2009;16(10):2738-2743.

19. Liu X, Sun X, Liu J, et al. Preoperative C-reactive protein/albumin ratio predicts prognosis of patients after curative resection for gastric cancer. Transl Oncol. 2015;8(4):339-345.

20. Ying HQ, Deng QW, He BS, et al. The prognostic value of preoperative NLR, d-NLR, PLR and LMR for predicting clinical outcome in surgical colorectal cancer patients. Med Oncol. 2014;31(12):305.

21. Hu B, Yang XR, Xu Y, et al. Systemic immune-inflammation index predicts prognosis of patients after curative resection for hepatocellular carcinoma. Clin Cancer Res. 2014;20(23):6212-6222.

22. Fairclough E, Cairns E, Hamilton J, Kelly C. Evaluation of a modified early warning system for acute medical admissions and comparison with C-reactive protein/albumin ratio as a predictor of patient outcome. Clin Med (Lond). 2009;9(1):30-33.

23. Liaw A, Wiener M. Classification and regression by random forest. R News. 2002;2(3):18-22.

24. Fluss R, Faraggi D, Reiser B. Estimation of the Youden index and its associated cutoff point. Biom J. 2005;47(4):458-472.

25. Nozoe T, Iguchi T, Adachi E, Matsukuma A, Ezaki T. Preoperative elevation of serum C-reactive protein as an independent prognostic indicator for gastric cancer. Surg Today. 2011;41(41):510-513.

26. Lee S, Oh SY, Kim SH, et al. Prognostic significance of neutrophil lymphocyte ratio and platelet lymphocyte ratio in advanced gastric cancer patients treated with FOLFOX chemotherapy. BMC Cancer. 2013;13(1):350.

27. Sun J, Chen X, Gao P, et al. Can the neutrophil to lymphocyte ratio be used to determine gastric cancer treatment outcomes? A systematic review and meta-analysis. Dis Markers. 2016;2016:7862469.

28. Songun I, Putter H, Kranenbarg EM, Sasako M, De Velde CJHV. Surgical treatment of gastric cancer: 15-year follow-up results of the randomised nationwide Dutch D1D2 trial. Lancet Oncol. 2010;11(5):439-449.

29. Rohatgi PR, Yao JC, Hess KR, et al. Outcome of gastric cancer patients after successful gastrectomy influence of the type of recurrence and histology on survival. Cancer. 2006;107(11):2576-2580.

30. Mantovani A, Allavena P, Sica A, Balkwill F. Cancer-related inflammation. Nature. 2008;454(7203):436-444.

31. Balkwill F, Mantovani A. Inflammation and cancer: back to Virchow? Lancet. 2001;357(9255):539.

32. Coussens LM, Werb, Z. Inflammation and cancer. Nature. 2002; 420(6917):860-867.

33. Moorthy K, Mohamed MS. Random forest for gene selection and microarray data classification. Bioinformation. 2010;7(3):142-146.

34. Chen XW, Liu M. Prediction of protein-protein interactions using random decision forest framework. Bioinformatics. 2005;21(24):4394-4400.

Cancer Management and Research

Publish your work in this journal

Cancer Management and Research is an international, peer-reviewed open access journal focusing on cancer research and the optimal use of preventative and integrated treatment interventions to achieve improved outcomes, enhanced survival and quality of life for the cancer patient. The manuscript management system is completely online and includes a very quick and fair peer-review system, which is all easy to use. Visit http://www.dovepress.com/testimonials.php to read real quotes from published authors.

\section{Dovepress}

Submit your manuscript here: https://www.dovepress.com/cancer-management-and-research-journal 